\title{
SELETIVIDADE E EFICÁCIA DA APLICAÇÃO SEQUENCIAL DE OXYFLUORFEN E DE IOXYNIL-OCTANOATO, EM SEMEADURA DIRETA DE CEBOLA ${ }^{1}$
}

\author{
LINO R. FERREIRA ${ }^{2}$, JULIO C. DURIGAN ${ }^{3}$, MANUEL G. C. CHURATA-MASCA ${ }^{3}$, FRANCISCO A. FERREIRA ${ }^{2}$ e \\ ANTÔNIO A. SILVA ${ }^{2}$
}

\begin{abstract}
Visando avaliar os efeitos do parcelamento da dose de oxyfluorfen e ioxyniloctanoato sobre a tolerância de plantas de cebola 'Granex 33' e a eficiência no controle das plantas daninhas na cultura, instalada por semeadura direta, foram conduzidos dois experimentos, sendo um na presença de plantas daninhas e o outro na ausência das mesmas. Ioxynil-octanoato, quando aplicado em doses acima de 0,125 kg/ha, aos 19 DAS, foi menos seletivo para o cultivar Granex 33 do que o oxyfluorfen. O parcelamento da dose, em até três aplicações, aumentou a tolerância das plantas de cebola ao oxyfluorfen e
\end{abstract}

RESUMO

ao ioxynil-octanoato. Oxyfluorfen, nas doses avaliadas, controlou Galinsoga parviflora e Amaranthus hybridus, porém não controlou Lepidium virginicum e Coronopus didymus. Ioxynil-octanoato a $0,125 \mathrm{~kg} / \mathrm{ha}$, aos $19 \mathrm{DAS}$, mais duas aplicações de $0,25 \mathrm{~kg} / \mathrm{ha}$ aos 34 e 65 DAS, controlou as espécies daninhas presentes, com produção de bulbos semelhantes à testemunha capinada. Nenhum dos herbicidas influenciou a conservação pós-colheita dos bulbos até 120 dias de armazenamento.

Palavras chave: Allium cepa, planta daninha, herbicida, controle, tolerância.

\section{ABSTRACT}

\section{Selectivity and efficacy of the sequential application of oxyfluorfen and ioxynyl-octanoate in direct sowing of onion}

With the purpose of evaluating the splitting of the dose of oxyfluorfen and ioxynyloctanoato on the tolerance of the 'Granex 33' onion plants and the efficiency on the weed control at crop, installed by direct sowing, two experiment were carried out, one in the presence and the other in the absence of weeds. Ioxynyloctanoato, when applied in doses higher than $0.125 \mathrm{~kg} / \mathrm{ha}$, at $19 \mathrm{DAS}$, was less selective for the 'Granex 33' cultivar than oxyfluorfen. Splitting of the doses, up to three applications, increased the tolerance of onion plants toward both oxyfluorfen and ioxynyl-octanoato. Oxyfluorfen, at the evaluated doses, controlled Galinsoga parviflora and Amaranthus hybridus; however it did not control Lepidium virginicum and Coronopus didymus. Ioxynyl-octanoato at 0.125 $\mathrm{kg} / \mathrm{ha}$, at $19 \mathrm{DAS}$, plus two applications of 0.25 $\mathrm{kg} / \mathrm{ha}$ at 34 and 65 DAS, controlled the existing weeds, with production of bulbs similar to the hand-hold control. None of the herbicides influenced the bulbs post-harvest conservation up to 120 days of storage.

Key words: Allium cepa, weed, herbicide, control, tolerance.

\footnotetext{
${ }^{1}$ Recebido para publicação em 22/05/98 e na forma revisada em 05/07/99. Parte da Tese de Doutorado do primeiro autor.

${ }^{2}$ Professores do Dept ${ }^{\circ}$ de Fitotecnia da UFV; CEP: 36571-000, Viçosa/MG.

${ }^{3}$ Professores da FCAVJ/UNESP; CEP: $14870-000$, Jaboticabal/SP. 


\section{INTRODUÇÃO}

A cultura da cebola é muito sensível à interferência das plantas daninhas, em razão da disposição ereta das folhas cilíndricas, porte baixo e lento desenvolvimento inicial das plantas, características que proporcionam baixa capacidade de sombreamento do solo, com baixo poder competitivo, permitindo a germinação de plantas infestantes durante do ciclo cultural.

$\mathrm{Na}$ semeadura direta, a interferência das plantas daninhas é ainda mais drástica, uma vez que, durante o estabelecimento da planta no campo, a capacidade de competição da mesma é muito pequena (Vorobiov \& Vasets'kii, 1984, citados por Pitelli, 1987). A redução da produção de bulbos ocorre, principalmente, em função da redução do estande, do número, do tamanho e da duração média das folhas, reduzindo, com isso, o peso médio dos bulbos. Outras alterações fisiológicas citadas são a antecipação do florescimento (Casamayor et al., 1974, Ferreira et al., 1978) e bulbificação precoce (Shadbolt \& Holm, 1956). Oliveira \& Alvarenga (1991) encontraram redução de $43,5 \%$ no número de folhas, $63,43 \%$ no peso médio dos bulbos e $76,34 \%$ na produção comercial, quando houve infestação (Galinsoga parviflora, Amaranthus spinosus e Sonchus oleraceus, respectivamente com 45,70; 28,28 e 15,09 \% do total da infestação) durante todo ciclo da cebola proveniente de semeadura direta.

Embora existam vários herbicidas indicados para a cultura da cebola, na verdade o são apenas para as culturas implantadas através de mudas ou de bulbinhos. As plantas de cebola provenientes de semeadura direta têm se mostrado mais sensíveis aos herbicidas, principalmente aos latifolicidas, apresentando grande redução na produção, quando estes são utilizados nas doses indicadas para o método de transplantio. Oxyfluorfen e ioxynil-octanoato, em doses reduzidas, têm apresentado resultados promissores (Leal et al., 1985; Oliveira Junior et al., 1995 e 1997, Ferreira, 1997), porém insuficientes para manter a cultura no limpo até à colheita.

Considerando que a cebola tem um ciclo longo, todo ele sujeito à interferência das plantas infestantes, considerando também que nos experimentos realizados em Monte Alto (Ferreira, 1997) o parcelamento da dose (duas aplicações) de oxyfluorfen e ioxynil-octanoato aumentou a tolerância da cultura a estes herbicidas, conduziuse este trabalho com objetivo de avaliar os efeitos do fracionamento da dose desses dois herbicidas, em até três aplicações, sobre a tolerância da cultura e a eficácia dos mesmos no controle das plantas daninhas dicotiledôneas, durante o ciclo da cebola, no sistema de semeadura direta.

\section{MATERIAL E MÉTODOS}

Foram conduzidos dois experimentos, sendo um na ausência e outro na presença de plantas daninhas, no período de 08/05 a 07/10/96, num solo de textura argilosa, com 1,95\% de matéria orgânica, no município de Viçosa, MG, latitude sul de $20^{\circ} 45^{\prime}$, latitude oeste de $42^{\circ} 52^{\prime}$ e altitude de $650 \mathrm{~m}$. Todos os tratos culturais da semeadura até à colheita foram semelhantes para os dois experimentos, à exceção das capinas.

$\mathrm{O}$ delineamento experimental utilizado foi de blocos ao acaso, com quatro repetições, com quinze e dezesseis tratamentos, respectivamente para os Experimentos 1 e 2, conforme listados na Tabela 1. Cada parcela experimental foi constituída de um canteiro de 1,0 $\mathrm{m}$ de largura por $3,0 \mathrm{~m}$ de comprimento. O espaçamento entre canteiros foi de $0,3 \mathrm{~m}$. A semeadura do cultivar Granex 33 foi feita em 08/05/96, em quatro fileiras espaçadas de $0,25 \mathrm{~m}$ com uma densidade de 40 sementes por metro de sulco. A área útil foi composta pelas duas fileiras centrais, eliminado-se $0,5 \mathrm{~m}$ de cada extremidade. 
TABELA 1. Herbicidas, doses e seus respectivos fracionamentos, épocas de aplicação e estádios de desenvolvimento das plantas de cebola, avaliados no Experimento 1 e 2.

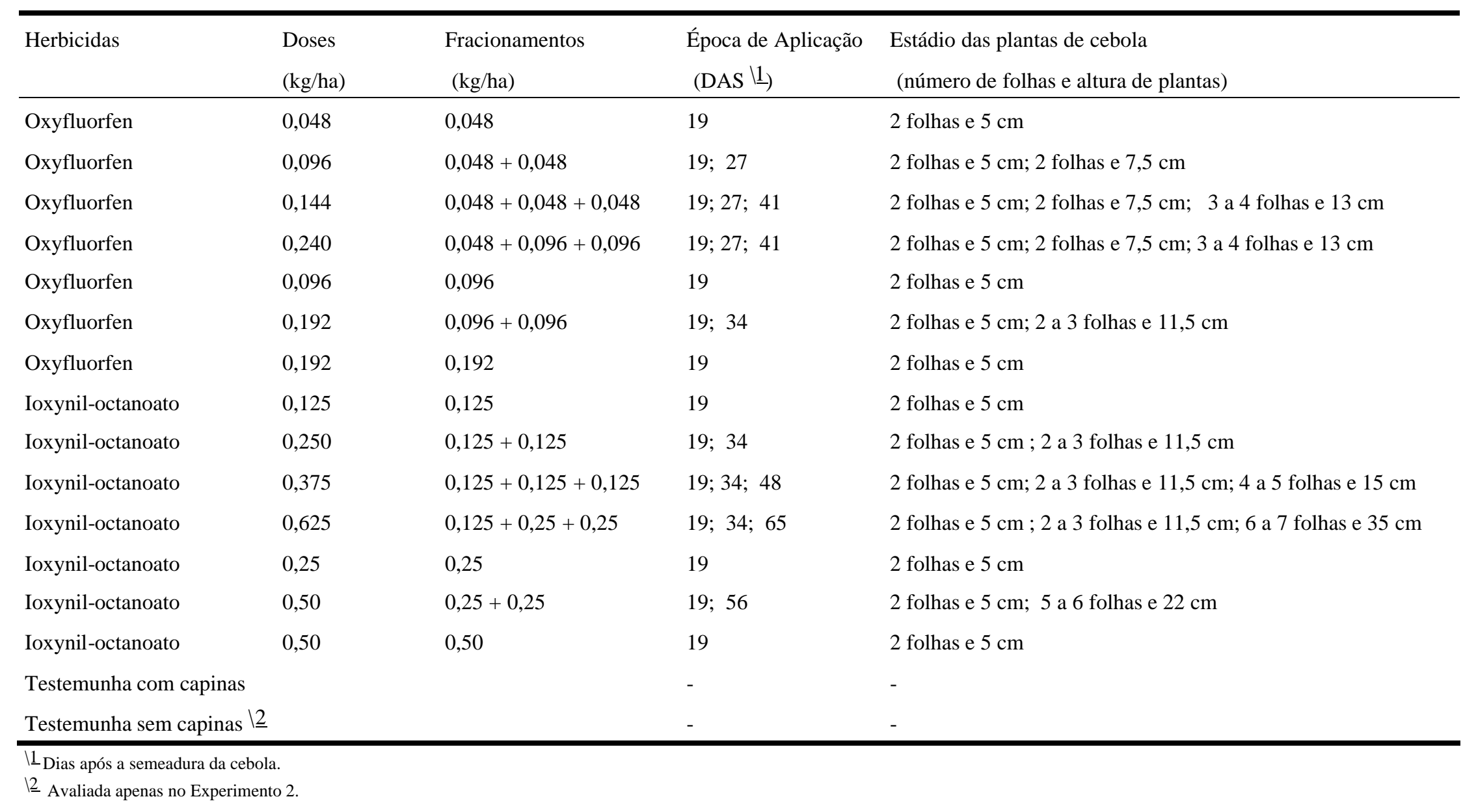


A adubação foi feita manualmente nos sulcos de semeadura utilizando-se $1000 \mathrm{~kg} / \mathrm{ha}$ de uma mistura composta por $63 \%$ de superfosfato simples, $18 \%$ de sulfato de amônio, $11 \%$ de cloreto de potássio, $7 \%$ de sulfato de magnésio, $0,5 \%$ de sulfato de zinco e $0,5 \%$ de bórax. Foram feitas quatro adubações em cobertura $(28,42,65$ e 82 DAS), cada uma com $120 \mathrm{~kg} / \mathrm{ha}$ de nitrocálcio.

Para aplicação dos herbicidas, foi utilizado um pulverizador costal com uma barra de dois bicos de jato plano ("leque") $110 \quad 03$ espaçados de $0,5 \mathrm{~m}$, à pressão constante de 2,1 $\mathrm{kgf} / \mathrm{cm}^{2}$, proporcionando volume de calda de 200 L/ha. A primeira dose dos herbicidas foi aplicada aos 19 DAS, quando as plantas de cebola estavam com duas folhas e, aproximadamente, $5 \mathrm{~cm}$ de altura e as plantas daninhas com até seis folhas. As demais aplicações foram feitas em função da reinfestação dos tratamentos observada no Experimento 2. Os estádios das plantas de cebola, na época de cada aplicação, estão apresentados na Tabela 1.

Todas as parcelas do Experimento 1 e a testemunha com capinas do Experimento 2 foram mantidas livres da interferência das plantas daninhas, a partir dos 20 DAS até à colheita, através de capinas manuais, realizadas quinzenalmente. As irrigações e pulverizações foram feitas de acordo com a necessidade da cultura.

No Experimento 1 foram avaliados o estande da cebola, aos 19, 26 e 41 DAS, a produção de bulbos e a capacidade de armazenamento dos mesmos. A colheita dos bulbos foi realizada aos 162 DAS, conforme recomendações técnicas para a cultura. Após a cura fez-se a limpeza, a classificação dos bulbos conforme o diâmetro horizontal e a pesagem dos mesmos (Brasil, 1982). Foram considerados para análise estatística a produção total, a produção e o peso médio dos bulbos comercializáveis (produção total excluindo os bulbos com diâmetro menor do que $20 \mathrm{~mm}$ ).

Para avaliar o efeito dos tratamentos sobre a conservação pós-colheita da cebola, foram colocados 10 bulbos de cada tratamento em sacos telados, num galpão ventilado por 120 dias. A cada 15 dias era feito uma pesagem, eliminando-se os bulbos podres e brotados. Aos 120 dias calculou-se a porcentagem de peso em relação ao peso inicial.

No Experimento 2, avaliou-se o número e a biomassa seca das plantas daninhas, separadas por espécie aos 77 DAS, amostrando-se uma área de $0,09 \mathrm{~m}^{2} /$ parcela e, através de avaliação visual, a porcentagem de cobertura com o total de plantas daninhas aos 33, 48, 61, 77, 101 e 162 DAS. Avaliou-se, também, a produção total, produção comercializável e o peso médio dos bulbos, seguindo os mesmos critérios descritos para o Experimento 1.

Fez-se a análise de variância dos tratamentos para as características avaliadas no dois experimentos. Em seguida as médias dos tratamentos foram comparadas pelo teste de Agrupamento de Scott-Knott a 5\% de probabilidade (Scott \& Knott, 1974).

\section{RESULTADOS E DISCUSSÃO}

\section{Experimento 1}

Não houve diferença entre as parcelas para o estande inicial, confirmando a boa uniformidade do mesmo, antes da aplicação dos herbicidas (Tabela 2). Os tratamentos com oxyfluorfen reduziram menos o estande de cebola do que com ioxynil-octanoato. Descontando-se a porcentagem ocorrida na testemunha $(2,5 \%)$, oxyfluorfen a $0,192 \mathrm{~kg} / \mathrm{ha}$, em aplicação única aos 19 DAS, proporcionou $22,6 \%$ de redução, ao passo que o ioxynil-octanoato nas doses de 0,250 e $0,500 \mathrm{~kg} / \mathrm{ha}$, ambas aplicadas aos 19 DAS, reduziu o estande em 35,6 e 80,2\%, respectivamente. As aplicações de doses menores aos 19 DAS, seguidas de doses maiores, foram os que menos reduziram o estande, para os dois herbicidas.

Todos os tratamentos com oxyfluorfen proporcionaram produções de bulbos (total e comercializável) semelhante à testemunha sem herbicidas, exceto para oxyfluorfen a $0,192 \mathrm{~kg} / \mathrm{ha}$, 
em uma (aos 19 DAS) ou duas aplicações ( aos 19 e 30 DAS). No caso do ioxynil-octanoato, todos tratamentos produziram menos que a testemunha, exceto quando foi parcelado em três aplicações, sendo uma de $0,125 \mathrm{~kg} / \mathrm{ha}$, aos 19 DAS e duas de $0,250 \mathrm{~kg} / \mathrm{ha}$, aos 34 e aos 65
DAS (Tabela 3). Maior peso médio de bulbos foi obtido com ioxynil-octanoato a $0,250 \mathrm{~kg} / \mathrm{ha} \mathrm{em}$ única aplicação aos 19 DAS, que também proporcionou a maior redução no estande, indicando boa capacidade de recuperação das plantas sobreviventes.

TABELA 2. Médias do estande inicial de cebola cv. Granex 33, aos 19 dias após a semeadura (DAS) e da porcentagem de redução do mesmo, aos 26 e 41 DAS, em função dos diferentes tratamentos. Experimento 1. Viçosa, MG, 1996.

\begin{tabular}{|c|c|c|c|}
\hline \multirow[t]{2}{*}{ Tratamentos (Doses em kg/ha e Épocas de Aplicação) } & \multirow{2}{*}{$\begin{array}{c}\begin{array}{c}\text { Estande Inicial } \\
(\text { planta/m) }\end{array} \\
19 \mathrm{DAS}\end{array}$} & \multicolumn{2}{|c|}{$\begin{array}{l}\text { Porcentagem de Redução } \\
\text { do Estande } \backslash 1\end{array}$} \\
\hline & & $26 \mathrm{DAS}$ & $41 \mathrm{DAS}$ \\
\hline Oxyfluorfen 0,048 (19 DAS) & 21,56 & $2,60 \mathrm{e}$ & $6,15 \mathrm{e}$ \\
\hline Oxyfluorfen $0,048(19$ DAS $)+0,048(27$ DAS $)$ & 21,31 & $4,66 \mathrm{e}$ & $9,36 \mathrm{e}$ \\
\hline Oxyfluorfen $0,048(19 \mathrm{DAS})+0,048(27 \mathrm{DAS})+0,048(41 \mathrm{DAS})$ & 18,44 & $4,13 \mathrm{e}$ & $15,09 \mathrm{e}$ \\
\hline Oxyfluorfen $0,048(19 \mathrm{DAS})+0,096(27 \mathrm{DAS})+0,096(41 \mathrm{DAS})$ & 21,75 & $2,66 \mathrm{e}$ & $11,68 \mathrm{e}$ \\
\hline Oxyfluorfen 0,096 (19 DAS) & 23,63 & $7,97 \mathrm{e}$ & $7,77 \mathrm{e}$ \\
\hline Oxyfluorfen 0,096 (19 DAS) + 0,096 (30 DAS) & 19,06 & $6,91 \mathrm{e}$ & $18,03 \mathrm{~d}$ \\
\hline Oxyfluorfen 0,192 (19 DAS) & 21,25 & $16,41 \mathrm{~d}$ & $25,12 \mathrm{~d}$ \\
\hline Ioxynil-octanoato 0,125 (19 DAS) & 21,56 & 6,43 e & $11,22 \mathrm{e}$ \\
\hline Ioxynil-octanoato $0,125(19 \mathrm{DAS})+0,125(34 \mathrm{DAS})$ & 23,37 & $16,22 \mathrm{~d}$ & $18,86 \mathrm{~d}$ \\
\hline Ioxynil-octanoato $0,125(19 \mathrm{DAS})+0,125(34 \mathrm{DAS})+0,125(48 \mathrm{DAS})$ & 18,69 & $7,22 \mathrm{e}$ & $13,61 \mathrm{e}$ \\
\hline Ioxynil-octanoato $0,125(19 \mathrm{DAS})+0,250(34 \mathrm{DAS})+0,250(65 \mathrm{DAS})$ & 20,87 & $9,88 \mathrm{e}$ & $20,70 \mathrm{~d}$ \\
\hline Ioxynil-octanoato 0,250 (19 DAS) & 18,69 & $33,14 \mathrm{c}$ & $38,15 \mathrm{c}$ \\
\hline Ioxynil-octanoato $0,250(19 \mathrm{DAS})+0,250(56 \mathrm{DAS})$ & 20,50 & $47,28 \mathrm{~b}$ & $57,07 \mathrm{~b}$ \\
\hline Ioxynil-octanoato 0,500 (19 DAS) & 18,25 & $63,47 \mathrm{a}$ & 82,80 a \\
\hline Testemunha com capinas & 19,87 & $0,65 \mathrm{e}$ & $2,55 \mathrm{e}$ \\
\hline C.V.(\%) & 15,28 & 35,32 & 34,12 \\
\hline
\end{tabular}

$\underline{1}$ Numa mesma coluna, as médias seguidas de mesma letra minúscula não diferem entre si, a $5 \%$ de probabilidade, pelo Teste de Agrupamento de Scott-Knott.

Nenhum dos herbicidas, em nenhum dos esquemas de fracionamento das doses, influenciou a conservação pós colheita dos bulbos, quando os mesmos foram armazenados em galpão ventilado, por até 120 dias (Tabela 3). As maiores perdas de peso observadas foram em função da grande capacidade de brotação do cultivar Granex 33 (dados não apresentados). Gomes (1987), também, não encontrou efeito sobre a conservação pós-colheita dos bulbos com aplicação de lactofen e de clorpropoxydim, em pós-emergência da cebola cv. Baia Periforme em semeadura direta.

\section{Experimento 2}

As principais plantas daninhas que ocorreram no local deste experimento foram: Galinsoga parviflora, Lepidium virginicum, Coronopus didymus e Amaranthus hybridus. Outras plantas ocorreram, porém em menor intensidade e de maneira muito desuniforme, como foi o caso de Sonchus oleraceus, Bidens pilosa, Ageratum conyzoides e Stachys arvensis. 
TABELA 3. Médias da produção total, da produção e do peso médio de bulbos comercializáveis, e da porcentagem de perda de peso, após 120 dias de armazenamento (DA), em relação ao peso inicial de cebola 'Granex 33', no sistema de semeadura direta, em função dos diferentes tratamentos. Experimento 1. Viçosa, MG, 1996.

\begin{tabular}{|c|c|c|c|c|}
\hline Tratamentos (Doses em kg/ha e Épocas de Aplicação) & $\begin{array}{l}\text { Prod. } \\
\text { Total } \backslash 1 \\
\text { (t/ha) }\end{array}$ & $\begin{array}{l}\text { Prod. } \\
\text { Com. } 11 \\
\text { (t/ha) }\end{array}$ & $\begin{array}{l}\text { Peso } \\
\text { Médio } \backslash 1 \\
\text { (g/bulbo) }\end{array}$ & $\begin{array}{l}\% \text { do Peso } \\
\text { Inicial } 1\end{array}$ \\
\hline Oxyfluorfen $0,048\left(19 \mathrm{DAS}^{12}\right)$ & $32,63 \mathrm{a}$ & $32,53 \mathrm{a}$ & $54,14 \mathrm{c}$ & 42,09 \\
\hline Oxyfluorfen $0,048(19 \mathrm{DAS})+0,048(27 \mathrm{DAS})$ & $31,04 \mathrm{a}$ & $30,79 \mathrm{a}$ & $55,92 \mathrm{c}$ & 40,13 \\
\hline Oxyfluorfen $0,048(19 \mathrm{DAS})+0,048(27 \mathrm{DAS})+0,048(41 \mathrm{DAS})$ & $30,72 \mathrm{a}$ & $30,60 \mathrm{a}$ & $62,41 \mathrm{c}$ & 38,68 \\
\hline Oxyfluorfen 0,048 (19DAS) + 0,096(27 DAS $)+0,096(41 \mathrm{DAS})$ & $31,11 \mathrm{a}$ & $31,00 \mathrm{a}$ & $58,21 \mathrm{c}$ & 40,90 \\
\hline Oxyfluorfen 0,096 (19 DAS) & $34,86 \mathrm{a}$ & $34,73 \mathrm{a}$ & $59,79 \mathrm{c}$ & 51,26 \\
\hline Oxyfluorfen 0,096 (19 DAS) + 0,096 (30 DAS) & $27,41 \mathrm{~b}$ & $27,32 \mathrm{~b}$ & $60,65 \mathrm{c}$ & 49,88 \\
\hline Oxyfluorfen 0,192 (19 DAS) & $26,53 \mathrm{~b}$ & $26,40 \mathrm{~b}$ & $58,06 \mathrm{c}$ & 38,57 \\
\hline Ioxynil-octanoato 0,125 (19 DAS) & $27,41 \mathrm{~b}$ & $27,24 \mathrm{~b}$ & $51,40 \mathrm{c}$ & 42,89 \\
\hline Ioxynil-octanoato $0,125(19 \mathrm{DAS})+0,125(34 \mathrm{DAS})$ & $26,95 \mathrm{~b}$ & $26,78 \mathrm{~b}$ & $51,12 \mathrm{c}$ & 37,20 \\
\hline Ioxynil-octanoato $0,125(19 \mathrm{DAS})+0,125(34 \mathrm{DAS})+0,125(48 \mathrm{DAS})$ & $29,37 \mathrm{~b}$ & $29,20 \mathrm{~b}$ & $57,89 \mathrm{c}$ & 48,56 \\
\hline Ioxynil-octanoato $0,125(19 \mathrm{DAS})+0,250(34 \mathrm{DAS})+0,250(65 \mathrm{DAS})$ & $32,57 \mathrm{a}$ & $32,42 \mathrm{a}$ & $64,71 \mathrm{c}$ & 62,11 \\
\hline Ioxynil-octanoato 0,250 (19 DAS) & $21,64 \mathrm{c}$ & $21,49 \mathrm{c}$ & $73,80 \mathrm{~b}$ & 40,83 \\
\hline Ioxynil-octanoato $0,250(19 \mathrm{DAS})+0,250(56 \mathrm{DAS})$ & $17,39 \mathrm{c}$ & $17,39 \mathrm{c}$ & $67,18 \mathrm{c}$ & 33,97 \\
\hline Ioxynil-octanoato 0,500 (19 DAS) & $10,13 \mathrm{~d}$ & $10,12 \mathrm{~d}$ & $88,63 \mathrm{a}$ & 38,43 \\
\hline Testemunha capinada & $35,45 \mathrm{a}$ & $34,94 \mathrm{a}$ & $59,16 \mathrm{c}$ & 32,02 \\
\hline C.V. (\%) & 14,22 & 14,12 & 13,53 & 30,78 \\
\hline
\end{tabular}

Houve efeito de tratamentos para o número de plantas e a biomassa seca de todas as plantas daninhas avaliadas, exceto para biomassa de outras plantas daninhas (Tabelas 4 e 5). Tanto o oxyfluorfen, quanto o ioxynil-octanoato, em todos os esquemas de fracionamento, reduziram o número de plantas e a biomassa seca de $G$. parviflora, quando comparados com a testemunha, embora esta redução tenha sido menor com oxyfluorfen nas doses de 0,048 e 0,096 kg/ha, em única aplicação aos 19 DAS. Os menores números e, também, as menores biomassa seca de $L$. virginicum e $C$. didymus foram obtidas nos tratamentos com reaplicações de ioxyniloctanoato. Estas duas espécies não foram eficientemente controladas pelo oxyfluorfen, embora na dose de 0,048 kg/ha, aos 19 DAS, seguida de duas aplicações de 0,096 kg/ha, sendo uma aos 27 e outra aos 41 DAS, não tenha diferido dos melhores tratamentos para $L$. virginicum. Todos os esquemas de fracionamento, tanto do oxyfluorfen, quanto do ioxynil-octanoato, reduziram o número e a biomassa seca das plantas de $A$. hybridus, a exceção do ioxynil-octanoato a $0,125 \mathrm{~kg} / \mathrm{ha}$, em única aplicação aos 19 DAS. Resultados semelhantes foram obtidos para $A$. lividus, que, também, não foi controlado pelo ioxynil-octanoato a $0,125 \mathrm{~kg} / \mathrm{ha}$, aplicado ao 27 DAS (Oliveira Junior et al., 1995).

Considerando o total das plantas daninhas, os menores números e, também, as menores biomassas secas foram obtidos com os fracionamentos das doses de ioxynil-octanoato. Observa-se, na Tabela 5, que G. parviflora foi a espécie predominante, na testemunha sem capina, proporcionando maior biomassa seca, em torno de $86 \%$ da biomassa total. 
TABELA 4. Médias do número de plantas daninhas, aos 77 dias após a semeadura (DAS) da cebola 'Granex 33', em função dos diferentes tratamentos. Experimento 2, Viçosa, MG, 1996.

\begin{tabular}{|c|c|c|c|c|c|c|}
\hline \multirow{2}{*}{$\begin{array}{l}\text { Tratamentos } \\
\text { (Doses em kg/ha e Épocas de Aplicação) }\end{array}$} & \multicolumn{6}{|c|}{ Número de Plantas Daninhas $/ \mathrm{m}^{2}$ I/ } \\
\hline & $\begin{array}{l}\text { Galinsoga } \\
\text { parviflora }\end{array}$ & $\begin{array}{l}\text { Lepidium } \\
\text { virginicum }\end{array}$ & $\begin{array}{l}\text { Coronopus } \\
\text { didymus }\end{array}$ & $\begin{array}{l}\text { Amaranthus } \\
\text { hybridus }\end{array}$ & Outras & Total \\
\hline Oxyfluorfen 0,048 (19 DAS) & $44,93 \mathrm{~b}$ & 125,39 a & 229,93 a & $0,00 \mathrm{c}$ & $0,00 \mathrm{~b}$ & $420,98 \mathrm{a}$ \\
\hline Oxyfluorfen 0,048 (19 DAS) + 0,048 (27 DAS) & $23,71 \mathrm{~b}$ & 140,63 a & 413,62 a & $0,00 \mathrm{c}$ & $0,00 \mathrm{~b}$ & $635,55 \mathrm{a}$ \\
\hline Oxyfluorfen 0,048 (19 DAS) + 0,048 (27 DAS) + 0,048 (41 DAS) & $1,40 \mathrm{c}$ & $64,14 \mathrm{~b}$ & $515,24 \mathrm{a}$ & $0,00 \mathrm{c}$ & $0,00 \mathrm{~b}$ & $684,89 \mathrm{a}$ \\
\hline Oxyfluorfen 0,048 (19 DAS) + 0,096 (27 DAS) + 0,096 (41 DAS) & $0,00 \mathrm{c}$ & $56,35 \mathrm{~b}$ & 202,28 a & $0,00 \mathrm{c}$ & $0,00 \mathrm{~b}$ & $284,43 \mathrm{a}$ \\
\hline Oxyfluorfen 0,096 (19 DAS) & $50,77 \mathrm{~b}$ & $131,60 \mathrm{a}$ & $142,54 \mathrm{a}$ & $0,00 \mathrm{c}$ & $1,40 \mathrm{~b}$ & $407,14 \mathrm{a}$ \\
\hline Oxyfluorfen 0,096 (19 DAS) + 0,096 (30 DAS) & $0,00 \mathrm{c}$ & 200,57 a & 195,22 a & $0,00 \mathrm{c}$ & $0,00 \mathrm{~b}$ & $346,58 \mathrm{a}$ \\
\hline Oxyfluorfen 0,192 (19 DAS) & $1,40 \mathrm{c}$ & 158,76 a & 226,91 a & $0,00 \mathrm{c}$ & $1,40 \mathrm{~b}$ & $484,38 \mathrm{a}$ \\
\hline Ioxynil-octanoato 0,125 (19 DAS) & $31,54 \mathrm{~b}$ & 126,96 a & $93,20 \mathrm{~b}$ & $57,56 \mathrm{a}$ & $5,26 \mathrm{~b}$ & $336,96 \mathrm{a}$ \\
\hline Ioxynil-octanoato 0,125 (19 DAS) + 0,125 (34 DAS) & $6,95 \mathrm{c}$ & $52,50 \mathrm{~b}$ & $16,23 \mathrm{~b}$ & $9,87 \mathrm{~b}$ & $3,74 \mathrm{~b}$ & $102,73 \mathrm{~b}$ \\
\hline Ioxynil-octanoato 0,125 (19 DAS) + 0,125 (34 DAS) + 0,125 (48 DAS) & $5,26 \mathrm{c}$ & $54,11 \mathrm{~b}$ & $32,33 \mathrm{~b}$ & $0,00 \mathrm{c}$ & $3,74 \mathrm{~b}$ & $110,17 \mathrm{~b}$ \\
\hline Ioxynil-octanoato $0,125(19$ DAS $)+0,250(34$ DAS $)+0,250$ (65 DAS) & $1,40 \mathrm{c}$ & $0,00 \mathrm{~b}$ & $2,46 \mathrm{~b}$ & $1,40 \mathrm{c}$ & $0,00 \mathrm{~b}$ & $3,42 \mathrm{~b}$ \\
\hline Ioxynil-octanoato 0,250 (19 DAS) & $7,94 \mathrm{c}$ & $40,33 \mathrm{~b}$ & $22,25 \mathrm{~b}$ & $5,26 \mathrm{~b}$ & $1,40 \mathrm{~b}$ & $93,20 \mathrm{~b}$ \\
\hline Ioxynil-octanoato 0,250 (19 DAS) + 0,250 (56 DAS) & $1,40 \mathrm{c}$ & $8,93 \mathrm{~b}$ & $5,26 \mathrm{~b}$ & $1,40 \mathrm{c}$ & $0,00 \mathrm{~b}$ & $20,11 \mathrm{~b}$ \\
\hline Ioxynil-octanoato 0,500 (19 DAS) & $6,58 \mathrm{c}$ & $15,58 \mathrm{~b}$ & $29,09 \mathrm{~b}$ & $6,63 \mathrm{~b}$ & $8,50 \mathrm{~b}$ & $93,01 \mathrm{~b}$ \\
\hline Testemunha sem capinas & $234,51 \mathrm{a}$ & $53,22 \mathrm{~b}$ & $134,52 \mathrm{~b}$ & $43,32 \mathrm{a}$ & 29,86 a & $652,30 \mathrm{a}$ \\
\hline C.V. (\%) & 53,02 & 56,78 & 71,73 & 73,05 & 77,74 & 37,49 \\
\hline
\end{tabular}

${ }^{1 /}$ Numa mesma coluna, as médias seguidas de mesma letra não diferem entre si, a 5\% de probabilidade, pelo Teste de Agrupamento de Scott-Knott. 
TABELA 6. Médias da porcentagem de cobertura com o total de plantas daninhas, em diferentes dias após a semeadura (DAS) da cebola cv. Granex 33, em função dos diferentes tratamentos. Experimento 2, Viçosa, MG, $1996^{1}$.

\begin{tabular}{|c|c|c|c|c|c|c|c|}
\hline \multirow{2}{*}{$\begin{array}{l}\text { Tratamentos } \\
\text { (Doses em kg/ha e Épocas de Aplicação) }\end{array}$} & \multicolumn{7}{|c|}{ Porcentagem de Cobertura ${ }^{1 /}$} \\
\hline & 33 DAS & 48 DAS & $61 \mathrm{DAS}$ & 77 DAS & $101 \mathrm{DAS}$ & 115 DAS & 162 DAS \\
\hline Oxyfluorfen 0,048 (19 DAS) & $7,75 \mathrm{~b}$ & $27,50 \mathrm{~b}$ & $77,50 \mathrm{~b}$ & 92,50 a & 95,00 a & 98,75 a & $100,00 \mathrm{a}$ \\
\hline Oxyfluorfen 0,048 (19 DAS) + 0,048 (27 DAS) & $7,25 \mathrm{~b}$ & $20,00 \mathrm{c}$ & $57,50 \mathrm{c}$ & 76,25 a & $80,00 \mathrm{a}$ & 87,50 a & $100,00 \mathrm{a}$ \\
\hline Oxyfluorfen $0,048(19$ DAS $)+0,048(27$ DAS $)+0,048$ (41 DAS) & $6,25 \mathrm{~b}$ & $6,00 \mathrm{~d}$ & $31,50 \mathrm{~d}$ & $51,25 \mathrm{~b}$ & $65,00 \mathrm{~b}$ & $75,00 \mathrm{~b}$ & $100,00 \mathrm{a}$ \\
\hline Oxyfluorfen 0,048 (19 DAS $)+0,096(27$ DAS $)+0,096(41$ DAS $)$ & $1,75 \mathrm{c}$ & $1,50 \mathrm{e}$ & $16,87 \mathrm{~d}$ & $31,75 \mathrm{c}$ & $56,25 \mathrm{~b}$ & $60,00 \mathrm{~b}$ & $100,00 \mathrm{a}$ \\
\hline Oxyfluorfen 0,096 (19 DAS) & $3,75 \mathrm{c}$ & $15,75 \mathrm{c}$ & $60,00 \mathrm{c}$ & 83,75 a & 85,00 a & 95,00 a & $100,00 \mathrm{a}$ \\
\hline Oxyfluorfen 0,096 (19 DAS) + 0,096 (30 DAS) & $1,25 \mathrm{c}$ & $4,50 \mathrm{e}$ & $31,00 \mathrm{~d}$ & $47,50 \mathrm{~b}$ & $70,00 \mathrm{~b}$ & 85,00 a & $100,00 \mathrm{a}$ \\
\hline Oxyfluorfen 0,192 (19 DAS) & $3,00 \mathrm{c}$ & $11,00 \mathrm{~d}$ & $45,75 \mathrm{c}$ & $67,50 \mathrm{~b}$ & $75,00 \mathrm{~b}$ & 86,25 a & $100,00 \mathrm{a}$ \\
\hline Ioxynil-octanoato 0,125 (19 DAS) & $3,00 \mathrm{c}$ & $10,00 \mathrm{~d}$ & $61,25 \mathrm{c}$ & 78,75 a & 90,00 a & 98,75 a & $100,00 \mathrm{a}$ \\
\hline Ioxynil-octanoato 0,125 (19 DAS) + 0,125 (34 DAS) & $2,75 \mathrm{c}$ & $1,62 \mathrm{e}$ & $6,75 \mathrm{e}$ & $23,50 \mathrm{c}$ & $36,25 \mathrm{c}$ & $66,25 \mathrm{~b}$ & $100,00 \mathrm{a}$ \\
\hline Ioxynil-octanoato $0,125(19 \mathrm{DAS})+0,125(34 \mathrm{DAS})+0,125(48 \mathrm{DAS})$ & $2,50 \mathrm{c}$ & $2,25 \mathrm{e}$ & $1,75 \mathrm{e}$ & $5,75 \mathrm{~d}$ & $11,50 \mathrm{~d}$ & $18,75 \mathrm{~d}$ & 95,00 a \\
\hline Ioxynil-octanoato $0,125(19 \mathrm{DAS})+0,250(34 \mathrm{DAS})+0,250(65 \mathrm{DAS})$ & $3,25 \mathrm{c}$ & $0,25 \mathrm{e}$ & $1,00 \mathrm{e}$ & $0,37 \mathrm{~d}$ & $0,50 \mathrm{~d}$ & $1,75 \mathrm{~d}$ & $20,00 \mathrm{c}$ \\
\hline Ioxynil-octanoato 0,250 (19 DAS) & $0,25 \mathrm{c}$ & $1,37 \mathrm{e}$ & $7,00 \mathrm{e}$ & $22,00 \mathrm{c}$ & $36,75 \mathrm{c}$ & $58,75 \mathrm{~b}$ & $100,00 \mathrm{a}$ \\
\hline Ioxynil-octanoato 0,250 (19 DAS) + 0,250 (56 DAS) & $0,00 \mathrm{c}$ & $0,62 \mathrm{e}$ & $0,50 \mathrm{e}$ & $0,77 \mathrm{~d}$ & $2,12 \mathrm{~d}$ & $6,87 \mathrm{~d}$ & $35,00 \mathrm{~b}$ \\
\hline Ioxynil-octanoato 0,500 (19 DAS) & $0,00 \mathrm{c}$ & $0,37 \mathrm{e}$ & $1,00 \mathrm{e}$ & $7,75 \mathrm{~d}$ & $20,50 \mathrm{c}$ & $42,50 \mathrm{c}$ & $100,00 \mathrm{a}$ \\
\hline Testemunha sem capinas & $77,50 \mathrm{a}$ & $98,75 \mathrm{a}$ & $100,00 \mathrm{a}$ & $100,00 \mathrm{a}$ & $100,00 \mathrm{a}$ & $100,00 \mathrm{a}$ & $100,00 \mathrm{a}$ \\
\hline C.V. $(\%)$ & 35.41 & 39,60 & 39,30 & 29,41 & 25,03 & 20,38 & 3,40 \\
\hline
\end{tabular}

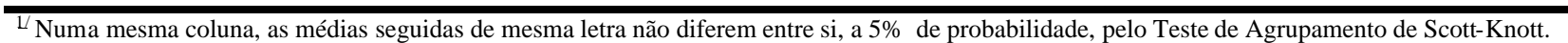


Em razão da população de plantas daninhas ser extremamente dinâmica e de $G$. parviflora ser controlado pelos dois herbicidas, em todos os esquemas de fracionamento da dose, houve maior produção de biomassa de $L$. virginicum, $C$. didymus e $A$. hybridus em alguns tratamentos, onde elas não foram eficientemente controladas, do que na testemunha sem capina.

Quanto a porcentagem de cobertura com o total das plantas daninhas (Tabela 6), houve efeito de tratamento para todas as épocas avaliadas. Observa-se, aos 33 DAS, que todos os tratamentos, à exceção da testemunha sem capina, apresentavam baixa cobertura foliar com plantas daninhas, indicando bom controle nesta época. A porcentagem de cobertura foi aumentando à medida que foram diminuindo os efeitos dos tratamentos aplicados. Observaram-se efeitos positivos das reaplicações dos herbicidas, sendo mais eficaz para o ioxynil-octanoato. Oxyfluorfen proporcionou maior porcentagem de cobertura, em razão da ineficiência no controle de $L$. virginicum e $C$. didymus. Na época da colheita, quando a maioria dos tratamentos apresentavam $100 \%$ de cobertura, ioxynil-octanoato a $0,125 \mathrm{~kg} / \mathrm{ha}$, aplicado aos 19 DAS, mais duas aplicações de $0,250 \mathrm{~kg} / \mathrm{ha}$, uma aos 34 e outra aos 65 DAS, apresentava apenas $20 \%$ de cobertura, ou seja $80 \%$ de controle do total das plantas infestantes. Observa-se, ainda, que ioxynil-octanoato a 0,500 $\mathrm{kg} / \mathrm{ha}$, aplicado de única vez aos 19 DAS, foi eficiente apenas até aos 101 DAS, não sendo eficaz para a colheita da cebola no limpo, concordando com resultados obtidos por Oliveira Jr. et al. (1995).

Apenas ioxynil-octanoato a $0,125 \mathrm{~kg} / \mathrm{ha}$, aplicado aos 19 DAS, mais duas aplicações de $0,250 \mathrm{~kg} / \mathrm{ha}$, uma aos 34 e outra aos 65 DAS, proporcionou produção (total e comercializável) e peso médio de bulbos semelhantes à testemunha com capinas. Os tratamentos com oxyfluorfen a $0,048 \mathrm{~kg} / \mathrm{ha}$, oxyfluorfen a $0,048+0,048 \mathrm{~kg} / \mathrm{ha}$, oxyfluorfen a $0,096 \mathrm{~kg} / \mathrm{ha}$, oxyfluorfen a $0,096+$ $0,096 \mathrm{~kg} / \mathrm{ha}$, oxyfluorfen a $0,192 \mathrm{~kg} / \mathrm{ha}$ e com ioxynil-octanoato a $0,125 \mathrm{~kg} / \mathrm{ha}$ não diferiram da testemunha sem capinas, quanto à produção de bulbos (Tabela 7). Esta redução na produção foi conseqüência da interferência das plantas daninhas com as plantas de cebola e, também em alguns casos, da toxicidade dos herbicidas. Comparando-se as produções dos Experimentos 1 e 2, os quais foram conduzidos, respectivamente, na ausência e presença das plantas daninhas podese afirmar que, para o oxyfluorfen a interferência das plantas daninhas foi a principal causa da redução na produção. Para o ioxynil-octanoato nas doses de 0,250 e $0,500 \mathrm{~kg} / \mathrm{ha}$, aplicadas aos 19 DAS, a redução do estande da cebola foi o fator mais importante, comprovando mais uma vez que, nas doses estudadas, o ioxynil-octanoato foi mais tóxico ao cultivar Granex 33 que o oxyfluorfen, estando de acordo com os resultados obtidos em Monte Alto, SP (Ferreira, 1997).

\section{LITERATURA CITADA}

BRASIL. Portaria $n^{\circ} 6305$ de 15 de dezembro de 1975. Aprova as normas de identificação, qualidade e embalagem de cebola. Decreto 8211, de 14 de agosto de 1978. Diário Oficial, Brasília, Seção 1, p. 7425-27, $1982 .$.

CASAMAYOR, C.R., PÉREZ, N.C., ACOSTA, C.O. Período crítico de competencia entre malas hierbas y la cebolla (Allium cepa L.). Cent. Agríc., Santa Clara, v.1, n.1, p.15-23, 1974.

FERREIRA, L.R. Eficácia e seletividade de herbicidas para a cultura da cebola (Allium cepa L.) em semeadura direta. Jaboticabal: UNESP, 1997, 130 p. Tese (Doutorado em Agronomia) - Universidade Estadual Paulista, 1997. 
TABELA 5. Médias da biomassa seca de plantas daninhas, aos 77 dias após a semeadura (DAS) da cebola Granex 33, em função dos diferentes tratamentos. Experimento 2. Viçosa, MG, 1996.

\begin{tabular}{|c|c|c|c|c|c|c|}
\hline \multirow{2}{*}{$\begin{array}{l}\text { Tratamentos } \\
\text { (Doses em kg/ha e Épocas de Aplicação) }\end{array}$} & \multicolumn{6}{|c|}{ Biomassa Seca ${ }^{1 /}\left(\mathrm{g} / \mathrm{m}^{2}\right)$} \\
\hline & $\begin{array}{l}\text { Galinsoga } \\
\text { parviflora }\end{array}$ & $\begin{array}{l}\text { Lepidium } \\
\text { virginicum }\end{array}$ & $\begin{array}{l}\text { Coronopus } \\
\text { didymus }\end{array}$ & $\begin{array}{l}\text { Amaranthus } \\
\text { hybridus }\end{array}$ & Outras & Total \\
\hline Oxyfluorfen 0,048 (19 DAS) & $76,86 \mathrm{~b}$ & $107,78 \mathrm{a}$ & 182,89 a & $0,00 \mathrm{~b}$ & 0,00 & $367,53 \mathrm{~b}$ \\
\hline Oxyfluorfen $0,048(19$ DAS $)+0,048$ (27 DAS) & $14,86 \mathrm{c}$ & 109,86 a & 205,47 a & $0,00 \mathrm{~b}$ & 0,00 & $357,97 \mathrm{~b}$ \\
\hline Oxyfluorfen $0,048(19$ DAS $)+0,048(27$ DAS $)+0,048$ (41 DAS $)$ & $1,00 \mathrm{c}$ & $70,22 \mathrm{a}$ & $197,44 \mathrm{a}$ & $0,00 \mathrm{~b}$ & 0,00 & $268,67 \mathrm{~b}$ \\
\hline Oxyfluorfen 0,048 (19 DAS) + 0,096 (27 DAS) + 0,096 (41 DAS) & $0,00 \mathrm{c}$ & $38,50 \mathrm{~b}$ & 143,78 a & $0,00 \mathrm{~b}$ & 0,00 & $182,28 \mathrm{c}$ \\
\hline Oxyfluorfen 0,096 (19 DAS) & $69,11 \mathrm{~b}$ & $124,67 \mathrm{a}$ & 151,69 a & $0,00 \mathrm{~b}$ & 5,92 & $351,39 \mathrm{~b}$ \\
\hline Oxyfluorfen 0,096 (19 DAS) + 0,096 (30 DAS) & $0,00 \mathrm{c}$ & $126,94 \mathrm{a}$ & 103,17 a & $0,00 \mathrm{~b}$ & 0,00 & $288,55 \mathrm{~b}$ \\
\hline Oxyfluorfen 0,192 (19 DAS) & $3,42 \mathrm{c}$ & 110,69 a & $213,50 \mathrm{a}$ & $0,00 \mathrm{~b}$ & 1,25 & $328,86 \mathrm{~b}$ \\
\hline Ioxynil-octanoato 0,125 (19 DAS) & $25,03 \mathrm{c}$ & $118,92 \mathrm{a}$ & 103,17 a & $58,08 \mathrm{a}$ & 15,39 & $320,58 \mathrm{~b}$ \\
\hline Ioxynil-octanoato 0,125 (19 DAS) + 0,125 (34 DAS) & $2,50 \mathrm{c}$ & $39,69 \mathrm{~b}$ & $20,50 \mathrm{~b}$ & $10,11 \mathrm{~b}$ & 23,69 & $96,50 \mathrm{c}$ \\
\hline Ioxynil-octanoato 0,125 (19 DAS $)+0,125(34$ DAS $)+0,125$ (48 DAS $)$ & $4,14 \mathrm{c}$ & $34,00 \mathrm{~b}$ & $18,42 \mathrm{~b}$ & $0,00 \mathrm{~b}$ & 6,03 & $88,55 \mathrm{c}$ \\
\hline Ioxynil-octanoato 0,125 (19 DAS $)+0,250$ (34 DAS $)+0,250$ (65 DAS) & $0,30 \mathrm{c}$ & $0,00 \mathrm{~b}$ & $1,25 \mathrm{~b}$ & $1,67 \mathrm{~b}$ & 0,00 & $1,72 \mathrm{c}$ \\
\hline Ioxynil-octanoato 0,250 (19 DAS) & $28,03 \mathrm{c}$ & $75,58 \mathrm{a}$ & $44,17 \mathrm{~b}$ & $1,53 \mathrm{~b}$ & 0,42 & $149,72 \mathrm{c}$ \\
\hline Ioxynil-octanoato 0,250 (19 DAS) + 0,250 (56 DAS) & $1,58 \mathrm{c}$ & $5,42 \mathrm{~b}$ & $1,30 \mathrm{~b}$ & $0,44 \mathrm{~b}$ & 0,00 & $8,75 \mathrm{c}$ \\
\hline Ioxynil-octanoato 0,500 (19 DAS) & $6,94 \mathrm{c}$ & $8,50 \mathrm{~b}$ & $39,14 \mathrm{~b}$ & $6,30 \mathrm{~b}$ & 5,08 & $65,97 \mathrm{c}$ \\
\hline Testemunha sem capinas & 484,25 a & $6,39 \mathrm{~b}$ & $47,92 \mathrm{~b}$ & $11,94 \mathrm{~b}$ & 10,72 & $561,22 \mathrm{a}$ \\
\hline C.V. $(\%)$ & 77,41 & 89,23 & 94,70 & 198,75 & 232,52 & 40,27 \\
\hline
\end{tabular}


TABELA 7. Médias da produção total, da produção e do peso médio de bulbos comercializáveis de cebola 'Granex 33', no sistema de semeadura direta, em função dos diferentes tratamentos. Experimento 2. Viçosa, MG, $1996^{1}$.

\begin{tabular}{|c|c|c|c|}
\hline Tratamentos (Doses em kg/ha e Épocas de Aplicação) & $\begin{array}{l}\text { Prod. } \\
\text { Total }^{1 /} \\
\text { (t/ha) }\end{array}$ & $\begin{array}{l}\text { Prod. } \\
\text { Comer. }{ }^{1 /} \\
(\mathrm{t} / \mathrm{ha})\end{array}$ & $\begin{array}{c}\text { Peso Médio }{ }^{1 /} \\
(\mathrm{g} / \mathrm{bulbo})\end{array}$ \\
\hline Oxyfluorfen $0,048\left(19\right.$ DAS $\left.^{2 /}\right)$ & $1,50 \mathrm{~d}$ & $0,32 \mathrm{e}$ & $6,25 \mathrm{e}$ \\
\hline Oxyfluorfen $0,048(19$ DAS $)+0,048(27$ DAS $)$ & $3,55 \mathrm{~d}$ & $2,78 \mathrm{e}$ & $12,73 \mathrm{e}$ \\
\hline Oxyfluorfen $0,048(19$ DAS $)+0,048(27$ DAS $)+0,048(41$ DAS $)$ & $7,75 \mathrm{c}$ & $6,80 \mathrm{~d}$ & $21,72 \mathrm{~d}$ \\
\hline Oxyfluorfen 0,048 (19 DAS $)+0,096(27$ DAS $)+0,096(41$ DAS $)$ & $10,68 \mathrm{c}$ & $10,05 \mathrm{~d}$ & $29,13 \mathrm{c}$ \\
\hline Oxyfluorfen 0,096 (19 DAS) & $1,87 \mathrm{~d}$ & $1,00 \mathrm{e}$ & $10,80 \mathrm{e}$ \\
\hline Oxyfluorfen 0,096 (19 DAS) + 0,096 (30 DAS) & $4,94 \mathrm{~d}$ & $4,26 \mathrm{e}$ & $18,79 \mathrm{~d}$ \\
\hline Oxyfluorfen 0,192 (19 DAS) & $2,89 \mathrm{~d}$ & $2,32 \mathrm{e}$ & $14,41 \mathrm{e}$ \\
\hline Ioxynil-octanoato 0,125 (19 DAS) & $1,21 \mathrm{~d}$ & $0,55 \mathrm{e}$ & $11,29 \mathrm{e}$ \\
\hline Ioxynil-octanoato $0,125(19 \mathrm{DAS})+0,125$ (34 DAS) & $15,39 \mathrm{~b}$ & $14,59 \mathrm{c}$ & $32,06 \mathrm{c}$ \\
\hline Ioxynil-octanoato $0,125(19 \mathrm{DAS})+0,125(34 \mathrm{DAS})+0,125(48 \mathrm{DAS})$ & $21,31 \mathrm{~b}$ & $20,95 \mathrm{~b}$ & $38,44 \mathrm{~b}$ \\
\hline Ioxynil-octanoato $0,125(19 \mathrm{DAS})+0,250(34 \mathrm{DAS})+0,250(65 \mathrm{DAS})$ & $31,83 \mathrm{a}$ & $31,60 \mathrm{a}$ & $63,09 \mathrm{a}$ \\
\hline Ioxynil-octanoato 0,250 (19 DAS) & $8,59 \mathrm{c}$ & $8,17 \mathrm{~d}$ & $31,59 \mathrm{c}$ \\
\hline Ioxynil-octanoato 0,250 (19 DAS) + 0,250 (56 DAS) & $17,68 \mathrm{~b}$ & $17,52 \mathrm{~b}$ & $49,09 \mathrm{~b}$ \\
\hline Ioxynil-octanoato 0,500 (19 DAS) & $7,72 \mathrm{c}$ & $7,43 \mathrm{~d}$ & $45,09 \mathrm{~b}$ \\
\hline Testemunha com capinas & $35,26 \mathrm{a}$ & $35,11 \mathrm{a}$ & $66,52 \mathrm{a}$ \\
\hline Testemunha sem capinas & $0,00 \mathrm{~d}$ & $0,00 \mathrm{e}$ & $0,00 \mathrm{e}$ \\
\hline C.V. $(\%)$ & 34,08 & 37,86 & 29,22 \\
\hline
\end{tabular}

FERREIRA, F.A., SILVA, J.F., SILVA, R.F. Controle químico de ervas daninhas na cultura da cebola (Allium cepa L.). Rev. Ceres, Viçosa, v.25, n.141, p.486-90, 1978.

GOMES, J.M. Controle de plantas daninhas na semeadura direta da cebola (Allium cepa L.). Viçosa, 1987, 60 p. Dissertação (Mestrado em Fitotecnia) - Universidade Federal de Viçosa.

LEAL, F.R., CHURATA-MASCA, M.G.C., DURIGAN, J.C., PITELLI, R.A. Controle de plantas daninhas na semeadura direta de cebola (Allium cepa L.). Rev. Ceres, Viçosa, v.32, n.179, p.63-74, 1985.

OLIVEIRA JUNIOR., R.S., SILVA, J.F., FERREIRA, L.R., REIS, F.P. Tolerância de três cultivares de cebola ao ioxyniloctanoato e oxyfluorfen em semeadura direta. Bragantia, v.54, n.2, p.335-42. 1995.

OLIVEIRA JUNIOR., R.S., SILVA, J.F., FERREIRA, L.R., REIS, F.P. Sensibilidade de cultivares de cebola (Allum cepa L.) implantados por semeadura direta ao oxyfluorfen aplicado em diferentes estádios de desenvolvimento da planta. Rev. Ceres, Viçosa, n.44, v.251, p.1-16, 1997.

OLIVEIRA, J.M.F., ALVARENGA, M.A.R. Efeito da competição de plantas daninhas em alguns parâmetros produtivos da cebola (Allium cepa L.) implantada através da semeadura direta. Ciênc. Prát., Lavras, v.15, n.1, p.32-42, 1991.

PITELLI, R.A. Efeitos de períodos de convivência e de controle das plantas daninhas no crescimento, nutrição 
mineral e produtividade da cultura da cebola (Allium cepa L.) Jaboticabal: UNESP, 1987. 140 p. Tese (Livre Docência) - Universidade Estadual Paulista, 1987.

SCOTT, A. .J. \& KNOTT, M. A cluster analysis method for grouping means the analysis of variance. Biometrics, North Carolina, $\mathrm{n}$. 30, p. 507-12, 1974.

SHADBOLT, C.A., HOLM, L.G. Some quantitative aspects of weed competition in vegetable crops. Weeds, Urbana, v.4, n.2, p.111-23, 1956. 